\title{
Framework for Adaptive Sampling of Point-Based Surfaces Using Geometry and Color Attributes
}

\author{
Duck Bong Kim ${ }^{1}$, Eui Chul Kang ${ }^{1}$, Kwan H. Lee ${ }^{1}$, and Renato B. Pajarola ${ }^{2}$ \\ ${ }^{1}$ Gwangju Institute of Science and Technology (GIST), \\ Intelligent Design and Graphics laboratory, Department of Mechatronics, \\ 1 Oryong-dong, Buk-gu, Gwangju, 500-712, Korea \\ \{eorka333, eckang, lee\}@kyebek.gist.ac.kr \\ ${ }^{2}$ Univ. of Zurich, Department of Informatics, Winterthurerstr. 190 \\ 8057, Zurich, Switzerland \\ pajarola@acm.org
}

\begin{abstract}
Point-based rendering has offered a powerful alternative to triangle meshes when it comes to the rendering of highly complex objects consisting of densely sampled point clouds due to its flexibility and simplicity. The technological advance of 3D scanners has made it possible to acquire color as well as geometry data of highly complex objects. However, scanning and acquisition systems often produce surfaces that are much more dense than actually required for the intended application. Mobile devices, computer games and distributed virtual environments must often operate on systems where rendering and transmission capacity is highly constrained and therefore require strict control over the level of detail used in models. In this research, we present a framework for adaptive sampling of point-based surfaces using both geometry and color information.
\end{abstract}

\section{Introduction}

The recent advancement of $3 \mathrm{D}$ scanners has made it possible to acquire color as well as geometry information of highly complex objects with very high speed and with good accuracy. However, the acquired data poses great challenges in storage, editing, transmission, and rendering due to the heavy data set. Therefore, simplification of highly detailed objects is necessary for the real time implementation and has become an important issue in many application fields such as entertainment, industrial design, virtual reality and other related fields.

Polygonal-based simplification has been studied by many researchers. In spite of the simplicity and flexibility of polygonal-based simplification, it has some limitations and drawbacks in some applications due to the need of establishing connectivity. However, a point-based method does not need the connectivity information during the simplification process. Efficient simplification of pointsampled surfaces has been researched $[1,2]$. However, many existing point-based simplification methods focus only on decimating an original model into simplified models considering only the geometry information. 


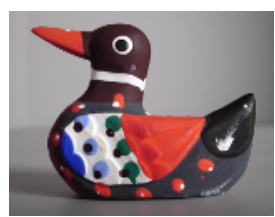

(a) Real object

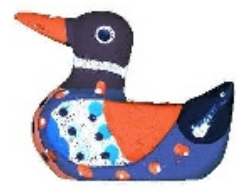

(b) Scanned data

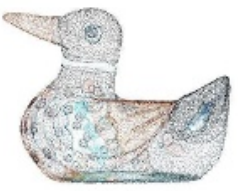

(c) Adapt. sampling

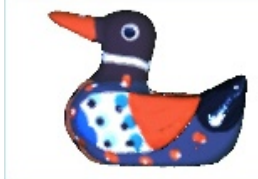

(d) Display

Fig. 1. 3D discrete LOD models of point-based surface creation pipeline

In our research, there are mainly three steps in adaptive sampling of the generation of point-based surfaces. Fig. 1 illustrates the whole process of generating 3D discrete LOD (level of detail) models of point-based surfaces acquired from a 3D scanning system. First, a real object is digitized using a 3D scanning device. The raw data from the 3D scanner is to be sampled adaptively according to the required level or desired application. Finally, the model is displayed using a point based rendering system based on blending and visibility splatting [3].

The main goal of this work is to present a framework for an adaptive sampling of point-based surfaces based on 3D grids using both color and geometry information to create the same visual quality and to speed up the rendering with lower sampling density.

\section{Adaptive Sampling of Point-Based Surface}

In this stage, the raw data from the $3 \mathrm{D}$ scanner is to be sampled adaptively according to the required level. The proposed method uses a $3 \mathrm{D}$ grid algorithm [4]. It subdivides the point cloud into a number of sub grids according to normal and color standard deviation of points, each of which is replaced by one representative point sample. Using the $3 \mathrm{D}$ grid algorithm, we can sample more points in the regions of high curvature and big color difference, as shown in Fig. $2(\mathrm{c})$ and $2(\mathrm{~d})$. Before using 3D grid algorithm, normal estimation is performed locally using an algorithm [3] based on covariance matrix for each point and its neighbors. Fig. 2 illustrates the whole procedure of the adaptive point sampling method based on 3D grids from the unorganized point data set. Fig. 2(a) shows the model Nefertiti. Initial grids are generated by a user defined input, as shown in Fig. 2(b). Each initial grid is subdivided according to the standard deviation of normal values, as shown in Fig. 2(c). The subdivided grids are repeatedly subdivided according to the standard deviation of color values, as shown in Fig. 2(d). A Refinement step is performed in order to avoid holes and intervention between discrete point samples, as shown in Fig. 2(e). Upon completion of the 3D grid subdivision, the decimated discrete point samples are converted into surface splats in order to fill the gap between the neighboring samples. In order to estimate the extent of each splat, another calculation is required. However, we estimate the extent of each splat according to the depth of each grid. 

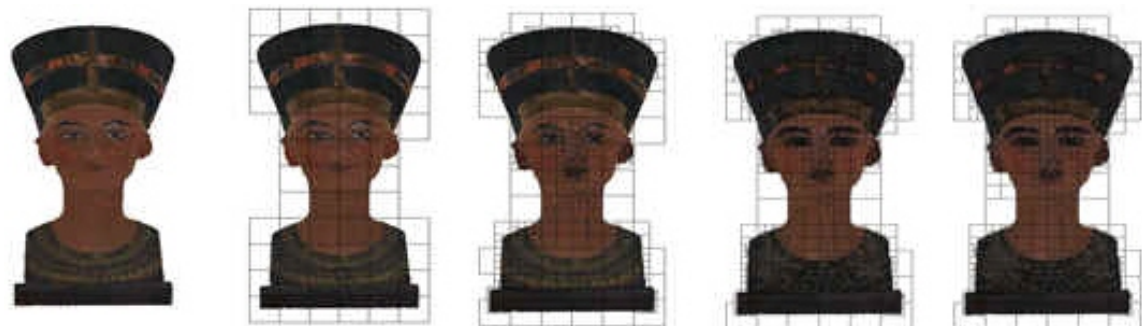

Fig. 2. Adaptive sampling method procedure: (a) Nefertiti (b) Initial grids (c) Subdivided by normal variation (d) Subdivided by color variation (e) Refinement

\section{Rendering Result}

Fig. 3 shows the visual quality comparison between the original model (left), the model simplified by uniform sampling method (left middle), the model simplified by only using geometry variation (right middle), and the model simplified by geometry and color variation (right). The model, Nefertiti, is simplified from the original point set of $448 \mathrm{k}$ to $14 \mathrm{k}$ by the uniform sampling method and the proposed algorithm and rendered by the proposed point-based rendering technique. This rendering result demonstrates that the eyes of Nefertiti simplified by geometry and color variation look sharp, while the eyes of Nefertiti, simplified by uniform sampling method and only geometry variation, are blurred.

Fig. 4 illustrates the visual quality according to 3D discrete LOD (level of detail) point-based surfaces. The visual quality of each point-based surface is poorer in accordance with the smaller 3D discrete LOD (level of detail) pointbased surfaces. However, the rendering speed time becomes faster as the level of detail decreases.
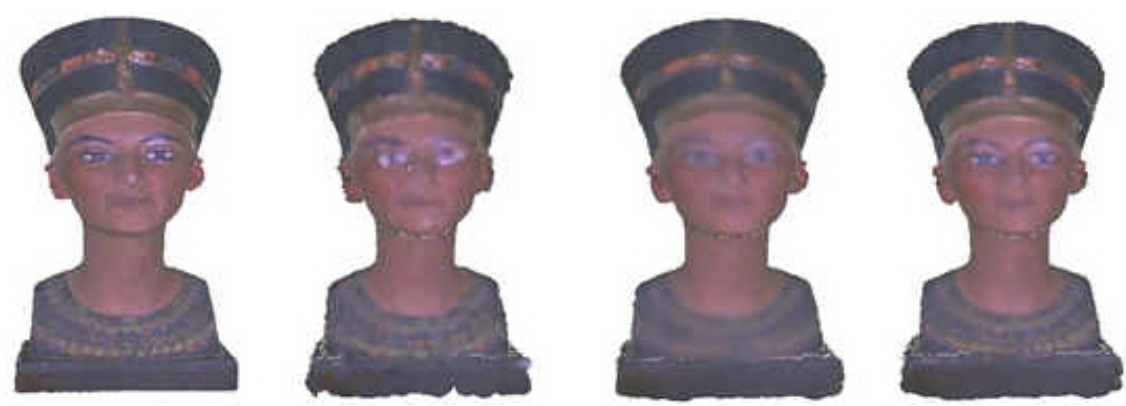

Fig. 3. Visual comparison of Model Nefertiti: (a) Original (b) sampled by uniform (c) using geometry variation (d) using geometry and color variation 

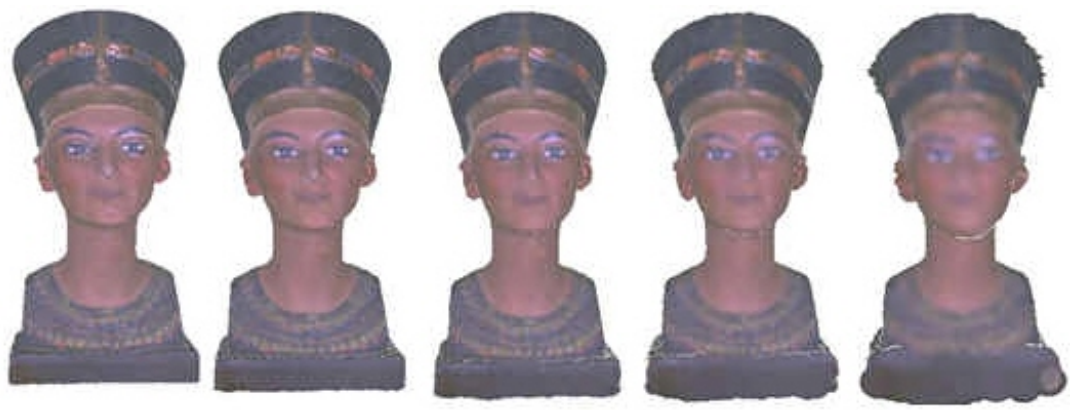

Fig. 4. Nefertiti at different levels of detail. From left to right, 450k, 110k, 45k, 14k and $5 \mathrm{k}$ points of the original model, rendered with a point splatting renderer.

\section{Conclusion}

In this paper, we proposed a framework for adaptive sampling of point-based surfaces using both geometry and color attributes. Experimental rendering results demonstrate that the visual quality of the model simplified by using geometry and color variation has the best quality compared with other approaches and a trade off exists between the visual quality and the speed of rendering time. In future we will define new mathematical criteria for determining the extent of each splat to generate point-based surfaces without holes and intervention.

\section{Acknowledgements}

This work was supported by the Immersive Contents Research Center and by the Realistic Broadcasting Research Center at GIST, Gwang-ju, South Korea and by the Korea Science and Engineering Foundation (KOSEF). The authors would like to thank Inus technology, Inc. for providing the geometric model Nefertiti.

\section{References}

1. M. Pauly, M. Gross, and L.P. Kobbelt, "Efficient Simplification of Point-Sampled Surfaces," Proc. IEEE Visualization 2002, pp. 163-170, 2002

2. J. Wu and L. Kobbelt, "Optimized Sub-Sampling of Point Sets for Surface Splatting", Computer Graphics Forum, 23(3), pp. 53-64. 2004

3. R.B.Pajarola, M.Sainz, P.Guidotiti. Confetti Object-Space Point Blending and Splatting. IEEE Transaction on Visualization and Computer Graphics, 2004

4. K. H. Lee, H. Woo and T. Suk, "Point Data Reduction Using 3D Grids", The International Journal of Advanced manufacturing Technology, pages 18:201-210, 2001 